\title{
A novel mutation in the steroidogenic acute regulatory protein gene promoter leading to reduced promoter activity
}

\author{
Andres J Casal, Victoria J P Sinclair ${ }^{1}$, Alessandro M Capponi, Jérôme Nicod ${ }^{1}$, \\ Uyen Huynh-Do ${ }^{1}$ and Paolo Ferrari ${ }^{1,2}$
}

Division of Endocrinology, Diabetology \& Nutrition, University Hospital, Geneva 1211, Switzerland

${ }^{1}$ Division of Nephrology, Inselspital, Berne 3010, Switzerland

${ }^{2}$ Department of Nephrology, Fremantle Hospital, University of Western Australia, Perth 6160, Australia

(Requests for offprints should be addressed to P Ferrari; Email: paolo.ferrari@ health.wa.gov.au)

\begin{abstract}
We have identified a novel cytosine/thymidine polymorphism of the human steroidogenic acute regulatory (StAR) gene promoter located $3 \mathrm{bp}$ downstream of the steroidogenic factor-1 (SF-1)-binding site and $9 \mathrm{bp}$ upstream of the TATA box (ATTTAAG). Carriers of this mutation have a high prevalence of primary aldosteronism. In transfection experiments, basal StAR promoter activity was unaltered by the mutation in murine $\mathrm{Y}-1$ cells and human H295R cells. In Y-1 cells, forskolin $(25 \mu \mathrm{M}, 6 \mathrm{~h})$ significantly increased wild-type promoter activity to $230 \pm 33 \%(P<0 \cdot 05, n=4)$. In contrast, forskolin increased mutated promoter activity only to $150 \pm 27 \%$, with a significant $35 \%$ reduction compared to wild type $(P<0 \cdot 05$, $n=3)$. In H295R cells, angiotensin II (Angll; $10 \mathrm{nM})$ increased wild-type StAR promoter activity to $265 \pm 22 \%(P<0.01$, $n=3)$, while mutated StAR promoter activity in response to Angll only reached $180 \pm 29 \%$ of controls $(P<0 \cdot 01, n=3)$. Gel mobility shift assays show the formation of two additional complexes with the mutated promoter: one with the transcription repressor DAX-1 and another with a yet unidentified factor, which strongly binds the SF-1 response element. Thus, this novel mutation in the human StAR promoter is critically involved in the regulation of StAR gene expression and is associated with reduced promoter activity, a finding relevant for adrenal steroid response to physiological stimulators.
\end{abstract}

Journal of Molecular Endocrinology (2006) 37, 71-80

\section{Introduction}

Recent reports suggest that variations at the gene CYP11B2 that encodes the aldosterone synthase enzyme are associated with essential hypertension, and may influence aldosterone secretion (Davies et al. 1999, Paillard et al. 1999, Takeda et al. 1999, Lim et al. 2002, Nicod et al. 2003). Two variants, a cytosine/thymidine $(\mathrm{C} / \mathrm{T})$ substitution in the $5^{\prime}$-promoter region, which disrupts a putative binding site for steroidogenic factor1 (SF-1), and a gene conversion in intron 2 (Int2 C) of CYP11B2 are in tight linkage disequilibrium (White \& Slutsker 1995, Lim et al. 2002, Nicod et al. 2003). This SF-1 T/Int2 C haplotype is increased in frequency in hypertensive patients (Davies et al. 1999) and has subsequently been associated with a high aldosteroneto-renin ratio (ARR) in selected hypertensive patients (Lim et al. 2002, Nicod et al. 2003). These findings indicate an increased sensitivity to angiotensin II (AngII) in the presence of the TT genotype of SF-1 in the promoter of CYP11B2.

The exact mechanisms for increased aldosterone synthase activity, associated with the $\mathrm{T}$ allele in the CYP11B2 promoter, are still objects of debate. Alternative explanations are increased transcription factor availability at other functional sites of the gene, linkage of the SF-1 and Int $2 \mathrm{C}$ sites with a quantitative trait locus in the regulatory elements or the possibility of transcriptional activation of the steroidogenic acute regulatory (StAR) gene by an SF-1-dependent mechanism (Garbers \& Dubois 1999). The latter is supported by the observation that only some, but not all, carriers of the $\mathrm{T}$ allele show increased aldosterone synthase activity.

Delivery of the substrate cholesterol to the inner mitochondrial membrane and to the cytochrome P450 side-chain cleavage enzyme is the rate-limiting step in steroid hormone synthesis (Crivello \& Jefcoate 1980, Privalle et al. 1983). The transfer of cholesterol from the outer mitochondrial membrane to the inner mitochondrial membrane, where it is cleaved to pregnenolone, is mediated by the StAR protein, which was identified and cloned by Clark et al. (1994). Mutations in the StAR gene are the cause of the potentially lethal condition known as congenital lipoid adrenal hyperplasia (lipoid CAH) (Lin et al. 1995). Mutations causing lipoid CAH are all found in the C-terminal region of the StAR protein (Miller 1997). StAR expression can be regulated both positively and negatively by agents that presumably act on its promoter (Stocco 2001). SF-1 was 
the first transcription factor to be studied as a potential regulator of the StAR gene. Several SF-1 consensusbinding sites have been identified in the StAR promoter (Caron et al. 1997, Sugawara et al. 1997). Two of these sites, located at positions -97 and -42 , are highly conserved in several species whose promoter regions have been sequenced. It is presently unknown whether polymorphisms at these sites are present and whether they might affect StAR promoter activity, thereby providing an explanation for the variable aldosterone synthase activity associated with the SF-1 polymorphism in the CYP11B2 promoter. Thus, in search of potential mechanisms underlying primary aldosteronism, we have screened the StAR gene for polymorphisms at SF-1-binding sites in hypertensive patients whose aldosterone and renin status was known.

\section{Materials and methods}

\section{Patients}

We screened for polymorphisms in the SF-1-binding sites of the human StAR gene promoter in 20 control subjects and 40 hypertensive patients, who had measurements of plasma renin and immunoreactive plasma aldosterone under standardised conditions as previously described (Nicod et al. 2003). Thereafter, the prevalence of the identified polymorphism was assessed in an additional cohort of 172 hypertensive patients who also had measurements of plasma immunoreactive renin and plasma aldosterone under standardised conditions and 76 healthy controls. Among all 212 hypertensive patients, 36 had an ARR above the diagnostic cut-off level for primary aldosteronism (Ferrari et al. 2004), but were not homozygous for the Tallele of CYP11B2, which is commonly associated with an increased ARR (Nicod et al. 2003). Finally, a group of 98 patients with diabetes mellitus type 2 was also tested for the presence of the same identified polymorphism. Thus, genotyping for the StAR gene promoter was performed on a total of 406 subjects.

\section{Screening for polymorphisms of the human StAR gene promoter}

The promoter region of the StAR gene harbouring the two conserved SF-1-binding sites and the TATA box was screened for the presence of polymorphisms by single strand conformation polymorphisms (SSCP) in 60 subjects using genomic DNA, isolated from peripheral blood leucocytes. The DNA (100-200 ng) was amplified in a $50-\mu \mathrm{l}$ reaction mixture containing $3 \mathrm{mM} \mathrm{MgCl}_{2}$, $0.4 \mu \mathrm{M}$ of each primer, $0.2 \mathrm{mM} \mathrm{dNTP}$ and $1 \mathrm{U}$ AmpliTaq Gold polymerase (Perkin Elmer Corp., Forster City, CA, USA) in the presence of the buffer provided with the enzyme. Thirty two cycles of PCR were performed: $30 \mathrm{~s}$ at $94{ }^{\circ} \mathrm{C}, 30 \mathrm{~s}$ at $62^{\circ} \mathrm{C}$ and $1 \mathrm{~min}$ at $72^{\circ} \mathrm{C}$. Primers were $5^{\prime}$-CTGTCCTCCCTACTCTCCCC$3^{\prime}$ and $5^{\prime}$-CTCTCAAGGGTGGTTCTTCG- $3^{\prime}$ and yielded a $258 \mathrm{bp}$ fragment amplifying the -328 to $-71 \mathrm{bp}$ region upstream to the start codon. All PCR products were analysed by SSCP on 12\% acrylamide gels containing $7 \cdot 25 \%$ glycerol using a two-buffer system. Four microlitres of the PCR sample were loaded and DNA was visualised by silver staining (Lovati et al. 2001). Any variations detected by this technique were characterised by directly sequencing the PCR amplified fragment with an ABI PRISM Model 3700 (Applied Biosystems, Foster City, CA, USA). This screening allowed us to identify a $\mathrm{C} / \mathrm{T}$ single nucleotide polymorphism (SNP) in the promoter region of the StAR gene, with loss of a MspA1 I restriction site in the mutant.

\section{Genotyping}

In the remaining sample of 366 subjects, genotyping of the identified SNP in the promoter of the StAR gene was done by specific restriction enzyme digestion. In all subjects, genomic DNA was amplified using the primers and conditions designed for the SSCP screening analysis. After amplification, the 258-bp long PCR fragments were digested for $2 \mathrm{~h}$ at $37^{\circ} \mathrm{C}$ with the MspA1 I restriction enzyme (New England Biolabs, Beverly, MA, USA), and the digestion products were separated by gel electrophoresis. The wild-type allele contains a recognition site for the restriction endonuclease so that digestion leads to two fragments of 103 and $155 \mathrm{bp}$. In the mutant allele, the recognition site is disrupted and therefore the amplified fragment is not digested.

\section{Plasmids and construction of StAR promoter mutants}

The wild-type pGL2-hStAR promoter construct was kindly provided by D Stocco (Texas Tech University, Lubbock, TX, USA). The mutated pGL2-hStAR bearing the $\mathrm{C} / \mathrm{T}$ substitution in the position $-33 \mathrm{bp}$ from the transcription start site was generated by using the QuickChange site-directed mutagenesis kit (Stratagene, Cedar Creek TX, USA) according to the instructions of the manufacturer. Mutagenic primers (StAR-mutprom-fwd 5'-GATGCACAGCCTTCAGTGGGGGACATTTAAGAC$3^{\prime}$ and StAR-mutprom-rev ${ }^{\prime} 5^{\prime}$-GTCTTAAATGTCCCCCACTGAAGGCTGTGCATC-3') were designed according to the GenBank sequence U29098. The mutated nucleotide is underlined. Four separate clones were obtained and tested, which gave comparable results in the reporter gene assays.

\section{Cell culture and transfections}

The human adrenocortical carcinoma NCI H295R cell line was kindly provided by Dr W E Rainey (University of 
Texas Southwestern Medical Center, Dallas, TX, USA) and maintained in a 1:1 mixture of Dulbecco's modified eagle medium (DMEM) and Ham's F12 medium containing pyridoxine, L-glutamine, and $15 \mathrm{mM}$ HEPES (Life Technologies). The culture medium was supplemented with $1 \%$ insulin, transferrin, selenium (ITS + , Becton Dickinson and Co. Labware, Bedford, MA, USA), and 2\% Ultroser (Ciphergen, Biosepra, France) as well as antibiotics (125 $\mathrm{\mu g} / \mathrm{ml}$ streptomycin and $125 \mathrm{IU} / \mathrm{ml}$ penicillin). Transfections were performed using the Nucleofector Transfection system (Amaxa, Cologne, Germany) according to the instructions of the manufacturer. Briefly, $5 \times 10^{6}$ cells were transfected with $3 \mu \mathrm{g}$ of either the wild type or the mutated pGL2-hStAR promoter construct. Cells were then plated on 12-well culture plates $\left(3 \times 10^{5}\right.$ cells/well $)$. Following $48 \mathrm{~h}$ of posttransfection, the cells were stimulated with various agents for the indicated periods of time in serum-free medium. Cells were then harvested and luciferase assay performed using the Dual-Luciferase Reporter Assay System (Promega). The pRLSV40 vector was used as an internal control to normalise transfection efficiency.

The mouse adrenocortical cancer cell line Y-1 was obtained from Cell Line Service (Heidelberg, Germany). The adherent cells were maintained in DMEM supplemented with $10 \%$ fetal calf serum and $200 \mathrm{nM}$ L-glutamine as well as with antibiotics as described elsewhere. The cells were plated on 12-well culture plates $\left(1.5 \times 10^{5}\right.$ cells/well) and transfections were performed using FuGENE 6 transfection reagent (Roche) according to the instructions of the manufacturer. Briefly, the cells were transfected with $1 \mu \mathrm{g}$ /well of either the wild type or the mutated pGL2-hStAR promoter construct. The pRLSV40 vector was used as an internal control to normalise transfection efficiency. Forty-eight hours after transfection, cells were stimulated with various stimuli for the indicated periods of time in serum-free media. Cells were then harvested and luciferase assay was performed as stated above. Both cell lines were grown at $37^{\circ} \mathrm{C}$ under an atmosphere of $5 \% \mathrm{CO}_{2}-95 \%$ air.

\section{Electrophoretic mobility shift assay (EMSA)}

Double-stranded DNA corresponding to nucleotides -42 to -24 of the human StAR promoter according to the sequence published by Sugawara et al. (1997), was $\left[\alpha^{32} \mathrm{P}\right]$ dATP-labelled by Klenow fill-in (Promega). Wildtype sense $5^{\prime}$-gatcAGCCTTCAGCGGGGGACAT-3' annealed with wild-type antisense $5^{\prime}$-gatcATGTCCCCCGCTGAAGGCT- ${ }^{\prime}$ and mutated sense $5^{\prime}$-gatcAGCCTTCAGTGGGGGACAT- $3^{\prime}$ annealed with mutated antisense $5^{\prime}$-gatcATGTCCCCCACTGAAGGCT-3'. Nuclear protein extracts $(7 \mu \mathrm{g})$ were incubated for $20 \mathrm{~min}$ at room temperature with 30000 c.p.m. of labelled probe and $2 \mu \mathrm{g}$ of poly[d(IC) ] in EMSA-binding buffer. The reaction mixture was loaded onto a non-denaturing $5 \%$ polyacrylamide gel for $1 \mathrm{~h} 30 \mathrm{~min}$ at $150 \mathrm{~V}$ in a cold room. Gels were dried and exposed to autoradiography films (Kodak and Sigma) for $72 \mathrm{~h}$. In supershift experiments, $1 \mu \mathrm{g}$ of purified rabbit polyclonal anti-chicken ovalbumin upstream promoter-transcription factor-I (COUP-TFI) (kindly provided by Dr M.L. Dufau, National Institutes of Health, NICHD, Behesda, MD, USA), anti-SF-1 (kindly provided by Prof. K Morohashi, Okasaki, Japan) or antiDAX-1 (kindly provided by Dr E. Lalli, Strasbourg, France) antibody was added to the mixture $30 \mathrm{~min}$ prior to the labelled probe. In chase experiments, 100fold excess of cold Sp1 (Santa Cruz Biotechnology, Santa Cruz, CA, USA) or SF-1 (5'-CAGCCTT-3') consensus sequence oligonucleotides were added to the mixture 30 min prior to the labelled probe.

\section{Analysis of data}

Results are expressed as means \pm s.E.M. The mean values were compared by ANOVA using Fisher's test. A value of $P<0.05$ was considered as statistically significant.

\section{Results}

\section{Identification of an SNP in the proximal StAR promoter}

The initial screening of the 40 hypertensive and 20 control subjects identified a potentially functional $\mathrm{C} / \mathrm{T}$ polymorphism located $33 \mathrm{bp}$ upstream from the transcription start site of the human StAR gene promoter, $3 \mathrm{bp}$ downstream of the SF-1 site and 9 bp upstream of the TATA box (Fig. 1). To date, this novel mutation is not described in public SNP databases (NCBI dbSNP or www.ensembl.org). The mutant allele was present in nine $(2 \cdot 2 \%)$ of the 406 subjects investigated. The prevalence of this novel mutation was $1 \cdot 3 \%(4 / 308)$ in the non-diabetic population. When this polymorphism was investigated in the 98 type 2 diabetics a prevalence of $5 \cdot 1 \%$ was found $\left(\chi^{2}=6 \cdot 27, P<0 \cdot 01\right.$ for diabetics versus non-diabetics). Four $(11 \cdot 1 \%)$ of the 36 hypertensive patients with raised ARR were carriers of the mutant $T$ allele of the StAR gene promoter.

\section{Reduced response of the mutated StAR promoter to cAMP messenger pathway activation in $\mathrm{Y}-1$ cells}

In order to determine whether the $\mathrm{C} / \mathrm{T}$ substitution affects StAR promoter activity, we transiently transfected murine adrenocortical Y-1 cells with either the wild type or the mutated StAR promoter cloned into the pGL2 vector containing firefly luciferase as reporter gene (Fig. 2). Basal promoter activity did not differ between the wild type and mutant promoter. As expected, 


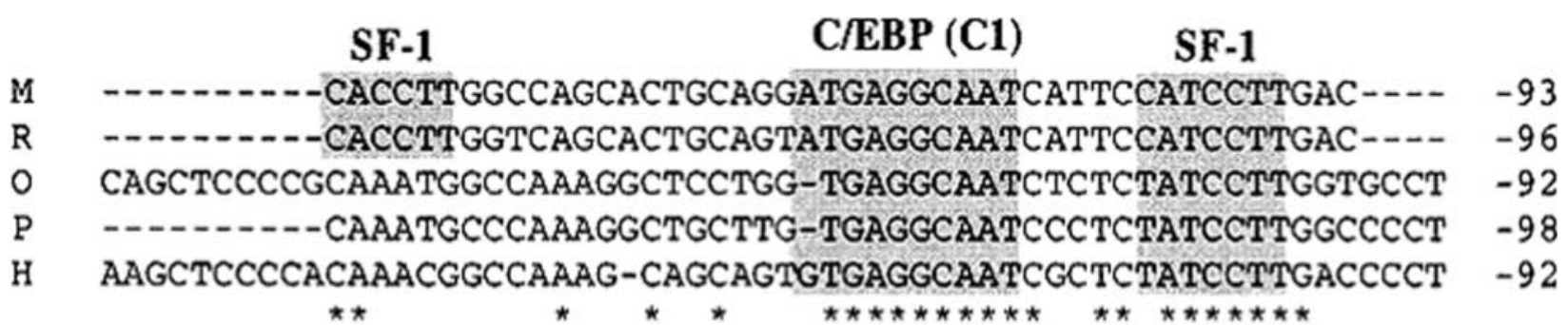

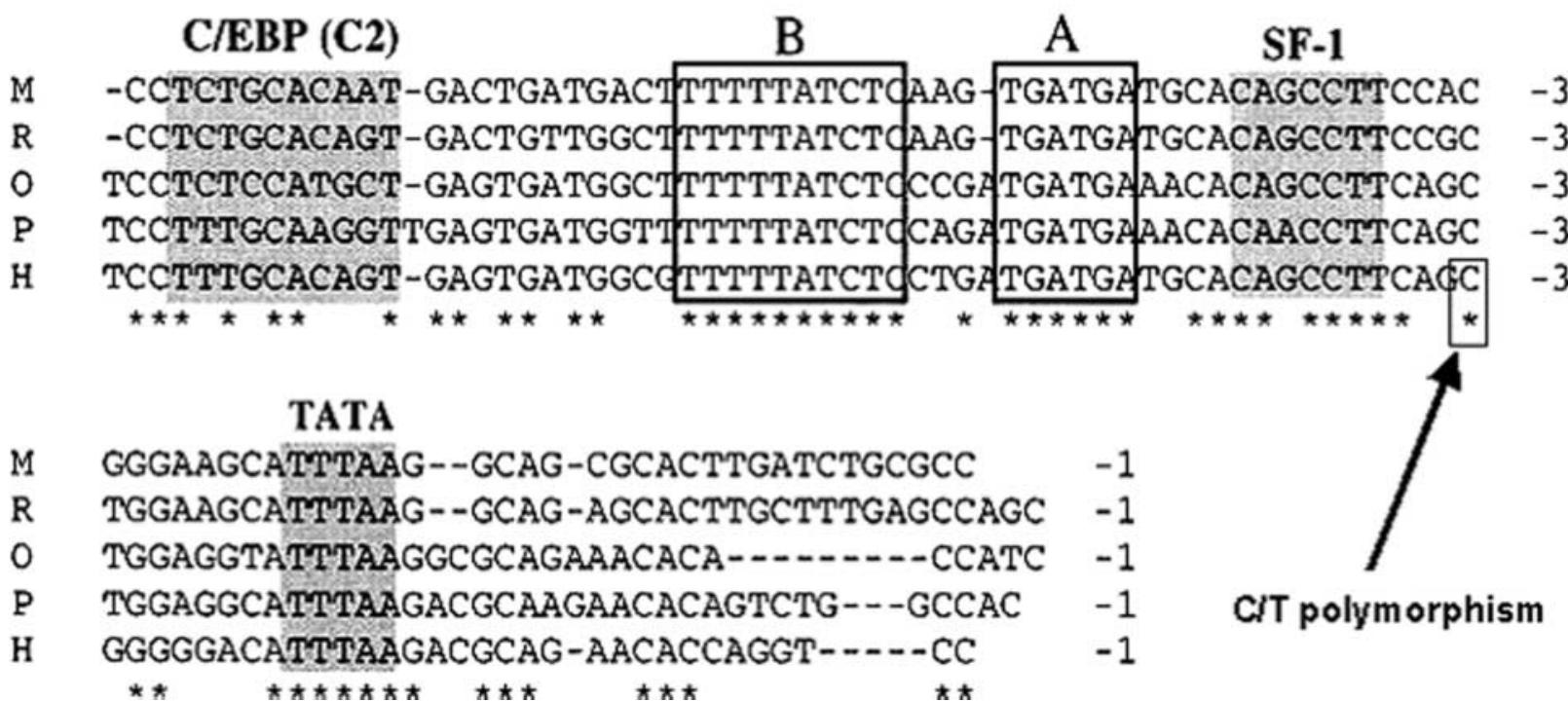

Figure 1 The sequences of the $5^{\prime}$-flanking regions of the mouse $(\mathrm{M})$, rat $(\mathrm{R})$, ovine $(\mathrm{O})$, porcine $(\mathrm{P})$, and human $(\mathrm{H})$ StAR genes are aligned, with identical bases in all five sequences indicated with asterisks. The SF-1 and C/enhancer binding protein-binding sites and the TATA box are shaded and labelled. Other highly conserved sequences are boxed. The localisation of the identified C/T polymorphism in the human StAR promoter at position -33 is indicated. (Modified with permission from Reinhart et al. 1999.)

forskolin treatment increased wild-type StAR promoter activity to $230 \pm 33 \%$ of control cells. In contrast, this response was markedly reduced with the mutated promoter, the maximal induction obtained with $25 \mu \mathrm{M}$ forskolin reaching only $150 \pm 27 \%$ of controls $(P<0 \cdot 05, n=3)$. This suggests that the $\mathrm{C} / \mathrm{T}$ substitution may play a crucial role in adequate StAR promoter transcriptional activity in response to activation of the cAMP messenger pathway.

Reduced response of the mutated StAR promoter to calcium messenger pathway activation in H295R cells

We then analysed whether the $\mathrm{C} / \mathrm{T}$ substitution also affects StAR promoter activity in response to activators of aldosterone biosynthesis. To this end, human H295R adrenocortical carcinoma cells were transiently transfected as above with either the wild type or the mutated StAR promoter cloned into the pGL2 vector (Fig. 3). As expected, AngII treatment increased wild-type StAR promoter activity to $265 \pm 22 \%$ of controls $(P<0 \cdot 01$, $n=3)$. In contrast, this response was markedly reduced with the mutated promoter, the maximal induction obtained with $10 \mathrm{nM}$ AngII reaching only $180 \pm 29 \%$ of controls $(P<0 \cdot 01, n=3)$. This suggests that the $\mathrm{C} / \mathrm{T}$ substitution may also play a crucial role in adequate StAR promoter transcriptional activity in response to activation of the calcium messenger pathway.

\section{Binding of various transcription factors to the mutated proximal StAR promoter}

EMSAs were performed in order to evaluate whether the mutation affects the interaction with the StAR promoter of various putative transcription factors known to be involved in the modulation of steroidogenesis, as compared with the wild-type StAR promoter. For this purpose we used a 23-bp DNA sequence spanning the mutation as well as the proximal SF-1 response element and half of the TATA box in the human StAR promoter as a probe for the experiment. 
$\star$

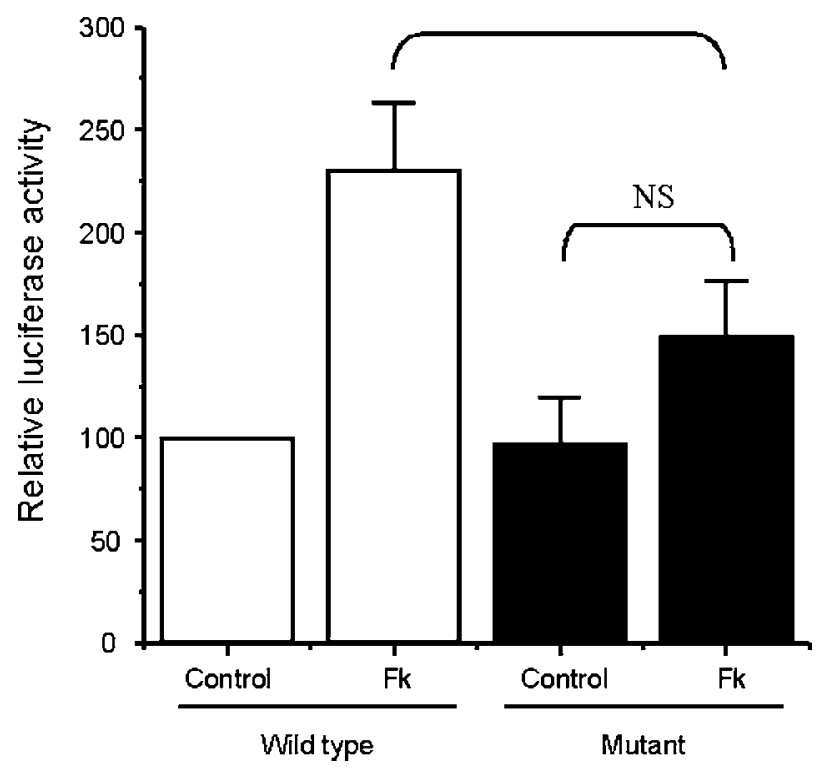

Figure 2 Effect of the mutation on StAR promoter activity in Y-1 mouse adrenocortical cells. Transcriptional activity of the StAR promoter was determined with a luciferase reporter system as described under 'Materials and methods'. Y-1 cells were transiently transfected with either the wild-type StAR promoter or the mutated StAR promoter cloned into the pGL2 vector containing firefly luciferase as a reporter gene. Renilla luciferase under control of the SV40 promoter was used as an internal control. Forty-eight hours after transfection, the cells were treated for $6 \mathrm{~h}$ with or without $25 \mu \mathrm{M}$ forskolin (Fk) and luciferase activity was determined. Results are expressed as percentage of control. Values are means \pm S.E.M. $(n=3$ separate experiments for each condition). ${ }^{\star} P<0.05$ vs control.

As expected from previous studies (Sugawara et al. 1997, Zazopoulos et al. 1997, Sandhoff et al. 1998), SF-1 and DAX-1, an activator and a repressor of StAR expression, respectively, specifically bound the StAR promoter probe, as demonstrated by supershifts induced with specific antibodies (Fig. 4A, lanes 2-3 and 6-7, and Fig. 4B, lanes 2-3 and 4-5). Interestingly, the band that was supershifted with the SF-1 antibody was also supershifted with a purified antibody directed against COUPTF (Fig. 4A, lanes $4-5$ and $4^{\prime}-5^{\prime}$ ) suggesting an interaction of COUP-TF with the StAR promoter and/or SF-1. These results were similar, whether we used the wild type or the mutated 23-bp probe. In contrast, the mutated probe induced an additional DAX-1 shift as demonstrated in supershift experiments using the DAX-1 antibody (Fig. 4B, lanes $2^{\prime}-3^{\prime}$ and $4^{\prime}-5^{\prime}$ ).

\section{Sp1 and SF-1 strongly bind to the mutated StAR promoter}

In order to further identify the additional DNA-protein interactions that occur on the mutated StAR promoter, we incubated nuclear extracts (NEs) incubated with

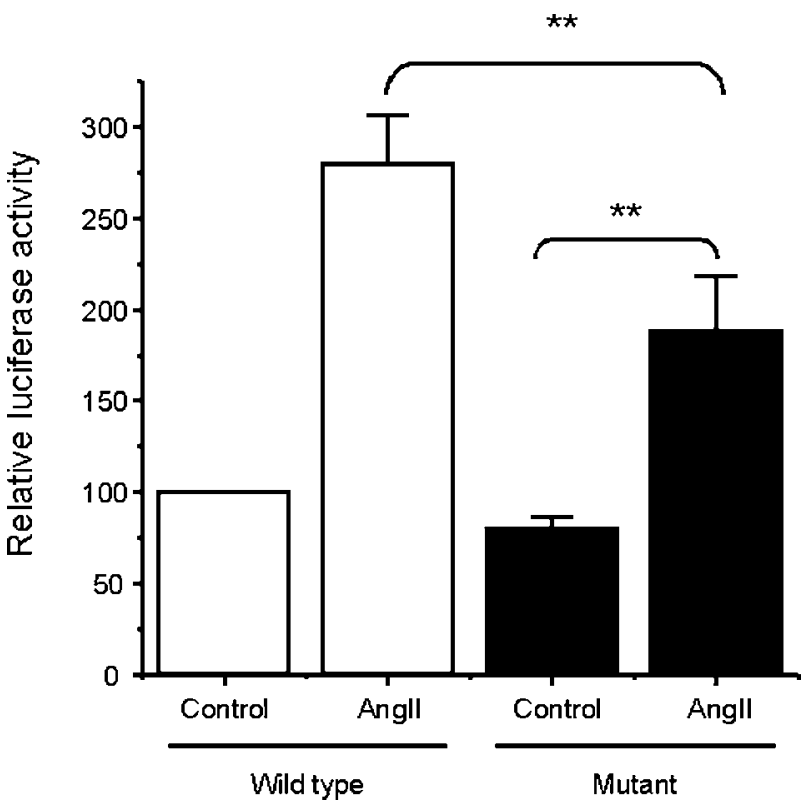

Figure 3 Effect of the mutation on StAR promoter activity in H295R human adrenocortical carcinoma cells. Transcriptional activity of the StAR promoter was determined with a luciferase reporter system as described under 'Materials and methods'. H295R cells were transiently transfected with either the wild-type StAR promoter or the mutated StAR promoter cloned into the pGL2 vector containing firefly luciferase as reporter gene. Renilla luciferase under control of the SV40 promoter was used as an internal control. Forty-eight hours after transfection, cells were treated for $6 \mathrm{~h}$ with $10 \mathrm{nM}$ Angll and luciferase activity was determined. Results are expressed as percentage of control. Values are means \pm S.E.M. $(n=3$ separate experiments for each condition). ${ }^{* \star} P<0.01$ vs control.

excess cold probe of the consensus response element for either Sp1 or SF-1, two transcription factors known to positively regulate the StAR gene (Sugawara et al. 2000) (Fig. 5).

As shown in Fig. 5A (left), the major shift observed when an NE of H295R cells was incubated in the presence of the labelled wild-type StAR promoter probe was abolished by an excess cold Sp1 oligonucleotide (lanes 4 and 5). In contrast, three of the four shifts observed with the mutated StAR promoter disappeared when a chase was performed with the Sp1 cold probe, suggesting that the mutation promotes the formation of an additional Sp1-DNA complex (Fig. 5A, lanes $4^{\prime}$ and $5^{\prime}$ ). The remaining protein-DNA complex observed with the mutant probe disappeared when competition was performed with a 100-fold excess of an SF-1 cold probe, indicating that the mutation promotes the reassembling of various crucial transcription factors over the proximal StAR promoter (Fig. 5B, lane 4). Also, the abundance of a slower migrating species was increased in the presence of excess unlabelled SF-1 oligonucleotide (Fig. 5B, lane 4). Although we have presently no explanation for this observation, it is 
A
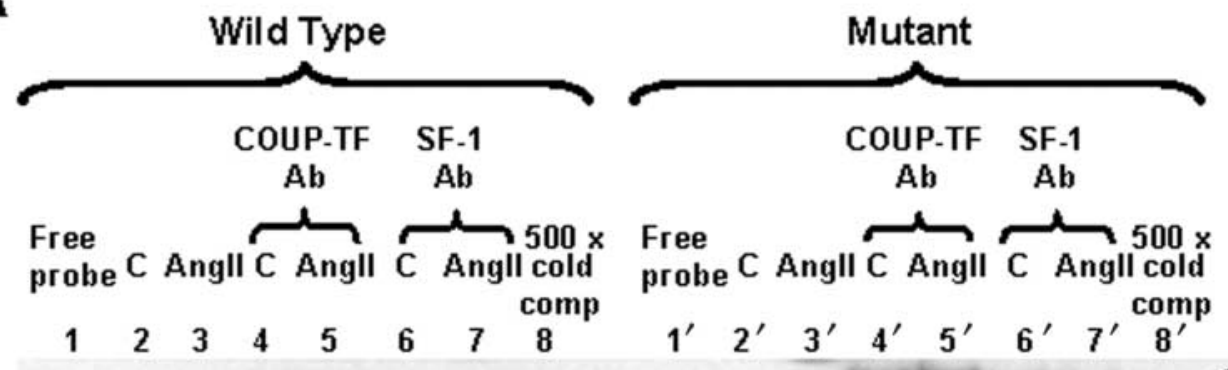

COUP-TF and SF -1 supershifts

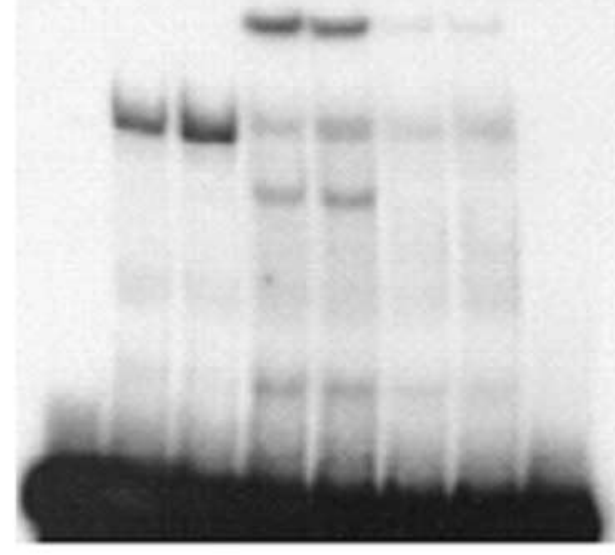

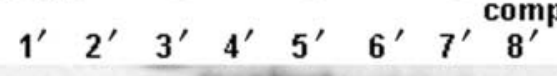
COUP-TF and SF-1 complex

B

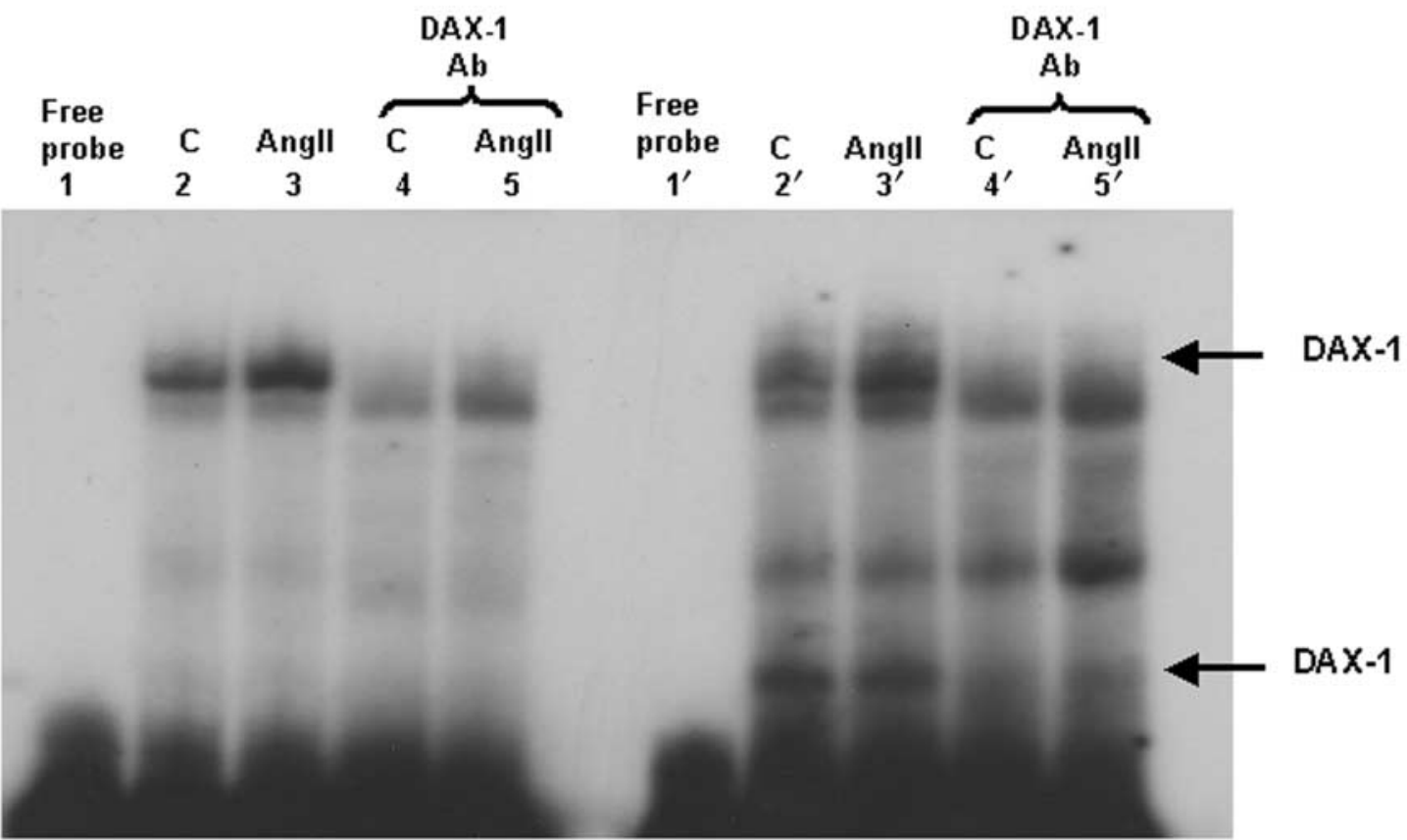

Figure 4 The $5^{\prime}$-flanking region of the mutated StAR promoter forms an additional DAX-1-DNA complex. (A) An oligonucleotide corresponding to region -42 to -24 of either the wild type or the mutated human StAR promoter and nuclear extract (NE) from H295R cells were used in EMSA experiments as described in 'Materials and methods'. Supershift assays were performed using specific antibodies (AB) against SF-1 and COUP-TF. (B) An EMSA with a specific DAX-1 antibody for supershift was performed with either the wild type or the mutated probe. Arrows indicate the bands corresponding to SF-1, to COUP-TF, to DAX-1 and to free probe. The figure shows a representative example from three separate experiments. C, control; Angll, angiotensin II. 


\section{A}
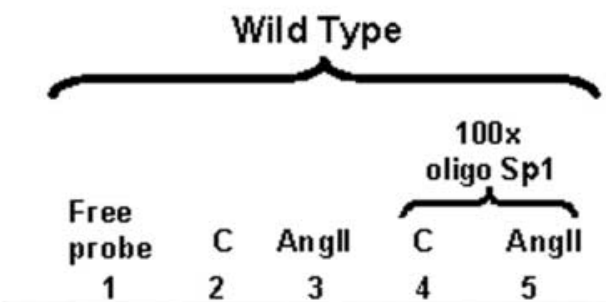
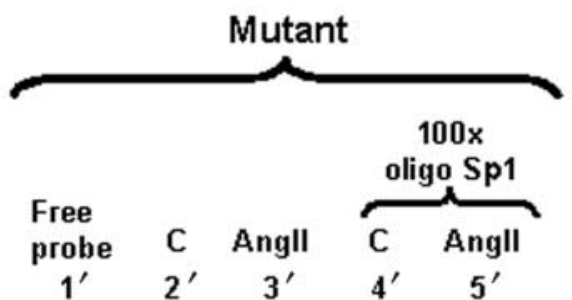

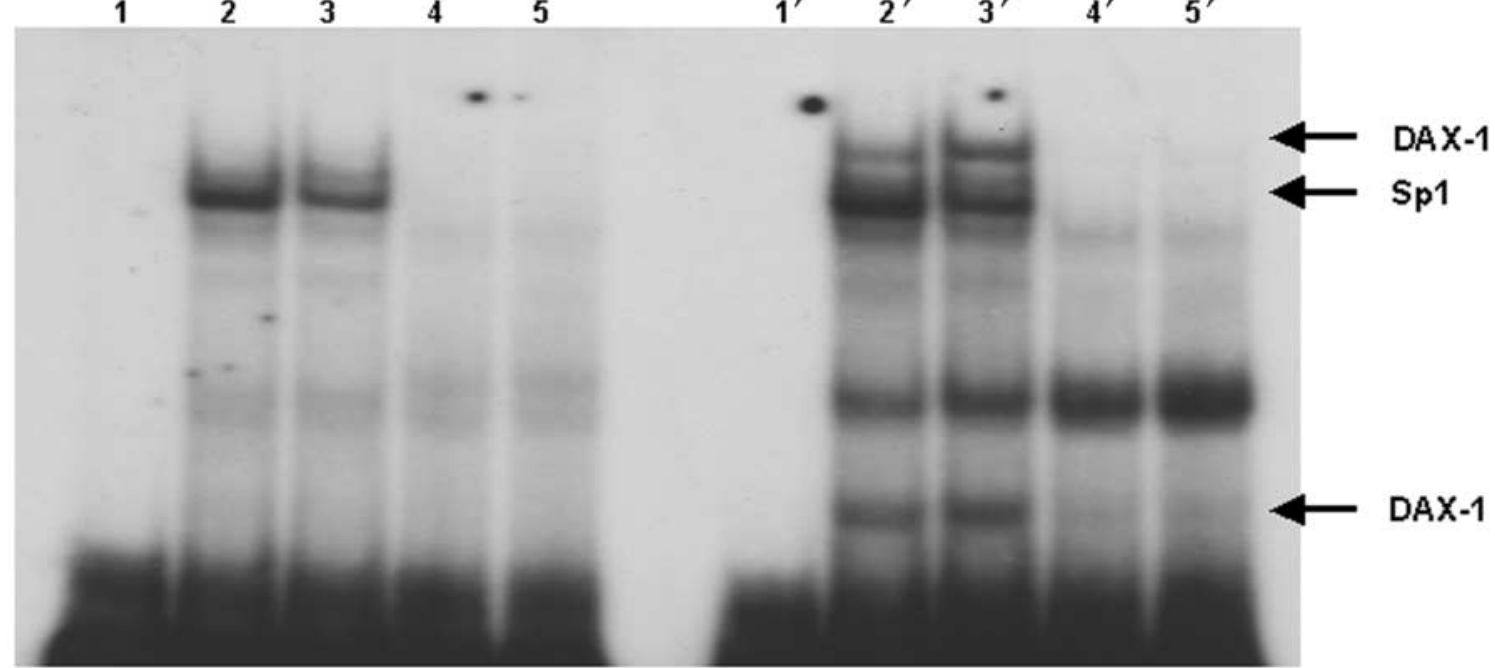

B

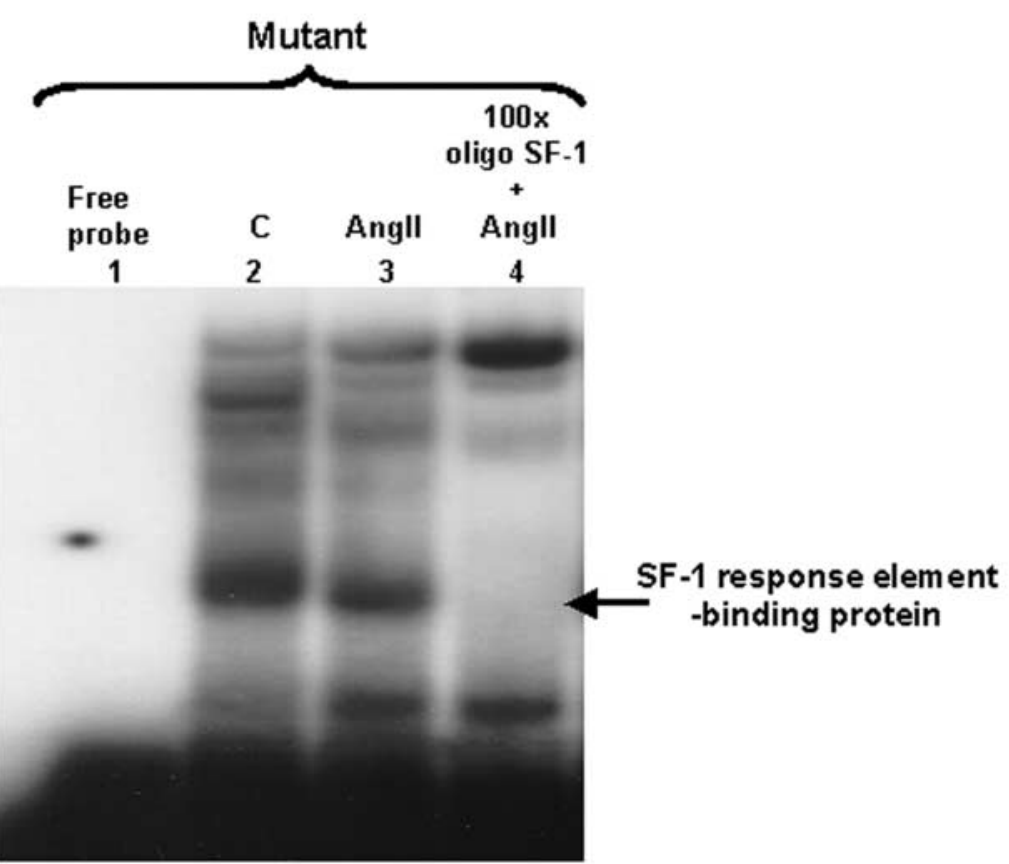

Figure 5 Binding of additional factor(s) to the SF-1 response element of the mutated StAR promoter. (A) Mobility shift assay was performed using a probe corresponding to region -42 to -24 of either the wild type or the mutated human StAR promoter incubated with NEs from H295R cells. Competition was performed with a 100-fold excess of cold Sp1 response element (GGCGGG). (B) The same gel shift experiment was performed using a 100-fold excess of cold SF-1 response element. C, control; Angll, angiotensin II. 
possible that, due to the availability of extra probe displaced by excess SF-1 oligonucleotide, there is increased binding of the probe to a non-specific complex.

\section{Discussion}

The search for functional polymorphisms in the promoter of the StARgene was undertaken to investigate potential mechanisms underlying primary aldosteronism in hypertensive patients with increased ARR not explained by the homozygosity of $-344 \mathrm{~T}$ allele at the SF1-binding site of CYP11B2 (Lim et al. 2002, Nicod et al. 2003). Homozygosity for the $-344 \mathrm{~T}$ allele of the CYP11B2 gene has been found to be associated with hypertension and increased aldosterone synthase activity as estimated by the ARR (Brand et al. 1998, Lim et al. 2002, Nicod et al. 2003). However, this genotype does not account for all subjects with raised ARR. Thus, it is possible that other proteins or enzymes involved in the cascade of aldosterone synthesis may be genetically altered to account for inadequately increased plasma aldosterone concentration for the prevailing circulating renin levels, in a subset of patients with primary aldosteronism. In the present study, 36 of 212 hypertensive patients had an ARR above the diagnostic cut-off level for primary aldosteronism (Ferrari et al. 2004). Genotyping of the StAR promoter showed that this novel StAR promoter mutation had a prevalence of $11.1 \%$ in these patients against the $1.7 \%$ prevalence in subjects with a normal ARR. On the other hand, $57 \cdot 1 \%$ of subjects with the mutation had an increased ARR.

Herein, we report a newly identified $\mathrm{C} / \mathrm{T}$ mutation located at position -33 from the transcription start site of the human StAR gene promoter, 3 bp downstream of the SF-1-binding site and $9 \mathrm{bp}$ upstream of the TATA box. To our knowledge, this is the first description of a mutation within the StAR promoter. This polymorphism lies in a crucial region for adequate StAR gene activity that bears a major response element for SF-1 (Sugawara et al. 2000). The identified mutation in the human StAR promoter is associated with a reduced promoter activity. As the region of the mutation is critically involved in the regulation of StAR gene expression, this finding may be relevant for adrenal steroid response to physiological stimulators.

Since the StAR protein mediates the crucial ratelimiting step of steroidogenesis, i.e. the transfer of cholesterol from the outer to the inner mitochondrial membrane, its expression must be finely regulated. The polymorphism we observed in the present study is located in a region of the StAR gene known to bear important response elements for the activation of steroidogenesis (Caron et al. 1997, Manna et al. 2003). This polymorphism is associated with the marked changes in promoter activity of the $1.3 \mathrm{~kb} 5^{\prime}$-flanking region of the StAR gene, as shown in two different adrenocortical cell lines, murine adrenocortical Y-1 cells and human H295R cells, with two different agonists.

Various mechanisms can concur to the observed reduction in StAR promoter activity. First, the mutation is located in close proximity to the most proximal response element for SF-1, an important-although not the sole-factor responsible for StAR gene expression (Manna et al. 2003). Secondly in the human promoter, the mutation is located within a nucleotide sequence which matches almost perfectly a GC box or consensus Sp1 box (GGCGGG, see Fig. 1) and Sp1 is a transcription factor known to activate the StAR gene by interacting with SF-1 (Sugawara et al. 2000). The presence of an Sp1binding site in this proximal region of the StAR promoter was confirmed by the fact that a consensus GC box was able to compete with the labelled oligonucleotide probe and chase it in EMSA assays (see Fig. 5A). It is, therefore, conceivable that the binding of Sp1 to its response element might be hampered in the mutated promoter and, as a consequence, the binding of SF-1 to its neighbouring response element. Thirdly the orphan nuclear receptor DAX-1, a repressor of StAR gene expression (Zazopoulos et al. 1997, Lalli et al. 1998, Osman et al. 2002), binds to a hairpin structure located at position -61 to -27 of the StAR promoter, a sequence which includes the present mutation. Zazopoulos and co-workers have shown that the binding of DAX-1 is stronger when the hairpin loop is rich in thymines (Zazopoulos et al. 1997). Interestingly, in the $\mathrm{C} / \mathrm{T}$ polymorphism we describe here, the hairpin loop is richer in thymines, a condition that should favour DAX1 binding to the promoter and repression of the StAR gene. Indeed, when DNA-protein interactions were analysed, gel shift assays revealed striking differences in the binding pattern of the probe (Fig. 4). As expected, SF-1 and DAX-1 specifically bound the StAR promoter probe. Both factors were shown to be involved in adequate StAR promoter activity (Caron et al. 1997, Sugawara et al. 1997, Osman et al. 2002). However, when NEs were incubated with the mutated probe, an additional DAX-1-DNA complex was formed. This result strongly supports the hypothesis suggesting increased binding of DAX-1 to a thymine-rich hairpin structure and thereby providing an explanation for the decrease in StAR promoter activity observed in the luciferase assays with the mutated construct.

In addition, this same additional complex also disappeared when competition was performed in the presence of a cold Sp1-response element. Since the sequence of the hairpin structure also includes the mutated Sp1 response element or GC box, these data suggest that the mutation may lead to decreased binding of Sp1, in addition to the increase in binding affinity of DNA for DAX-1. 
In contrast, basal activity was not reduced in the mutated promoter. This may be due to the fact that repression of the StAR promoter is already maximal in the resting state. Indeed, we have recently shown that both DAX-1 (Osman et al. 2002) and COUP-TF (Buholzer et al. 2005), two repressors of the StAR promoter, are robustly expressed in adrenal glomerulosa cells and that overexpression of either factor does not affect basal promoter activity.

The second additional protein-DNA complex formed with the mutated probe disappeared when the $\mathrm{NE}$ was incubated in the presence of an excess of cold SF-1 consensus sequence and of the labelled mutated probe (Fig. 5B). However, a similar supershift could not be obtained with a specific antibody directed against SF-1 (Fig. 4A), suggesting that the mutation induces the binding of an yet unidentified factor to the SF-1 response element.

In addition to binding specific DNA sequences, Sp1, SF-1 and DAX-1 interact with each other and promote or counteract each other's binding to the promoter, bringing an additional degree of complexity to the regulation of the StAR proximal promoter (Ito et al. 1997, Sugawara et al. 2000, Babu et al. 2002, Suzuki et al. 2003). It is, therefore, likely that the change in binding affinity of one factor for the StAR promoter resulting from the mutation may affect the binding of its partner to this same promoter. Moreover, recent studies have demonstrated that the regulatory actions of SF-1 and Sp1 may be mediated by components of the chromatin environment such as the ubiquitous co-activator CBP/ 300 or histone deacetylases (Monte et al. 1998, Zhang \& Dufau 2003, Hiroi et al. 2004). Thus, in vivo, the genomic environment may play an additional role in modulating the activity of the mutated StAR promoter.

Interestingly, a supershift was also observed with the antibody to COUP-TF for the same band that was also supershifted with the SF-1 antibody. This suggests an interaction with the StAR promoter and/or SF-1. COUP-TF is known to play a role as a repressor of steroidogenesis and, in particular, of StAR gene expression (Shibata et al. 2001, 2003, Buholzer et al. 2005). In addition, the competition between SF-1 and COUP-TF for a common binding site has been reported in the transcriptional regulation of the bovine CYP17 gene (Bakke \& Lund 1995) and modulation of the activity of the murine DAX-1 and aromatase P450 promoters (Zeitoun et al. 1999, Cooney et al. 2001). Our supershift assay results, however, do not suggest an involvement of COUP-TF in the effects induced by the mutation. Thus, we can conclude that a point mutation on the locus for a key regulator may induce a complete remodelling of the various transcription factors involved in the regulation of the StAR gene, and therefore, its activity.
We focused on the gene for the StAR protein because of its rate-limiting role in steroid biosynthesis and because an increased supply of steroid precursors could result in increased aldosterone production. Clearly, based on the in vitro expression studies, the functional effect of this novel mutation in the StAR promoter argues against the hypothesised mechanism. We would like to propose an alternative explanation for the present findings. The described StAR promoter mutation, the first of its kind, leads to reduced StAR promoter activity in response to adrenocorticotrophin (ACTH) and AngII. The main effect is likely to be a slight reduction in cortisol levels in response to ACTH. In turn, normal feedback regulation should result in a resetting of the hypothalamic-pituitary-adrenal axis such that cortisol levels are maintained. Consequently, there will be a subtle increase in the ACTH drive to the adrenal cortex. In the long term this is likely to cause hyperplasia of both zona fasciculata and zona glomerulosa of the adrenal cortex, resulting in increased synthetic capacity for both cortisol and aldosterone. In turn, this might lead to hypertension associated with altered regulation of aldosterone and possibly cause insulin resistance and increased predisposition to diabetes associated with altered cortisol secretion.

In view of the pleiotropic action of the StAR protein and because of the complex interactions between the various factors induced by this mutation, further experiments are needed to elucidate the exact role this polymorphism may play in states of altered function of the mineralocorticoid, glucocorticoid and sex hormone axes.

\section{Acknowledgements}

The authors are grateful to Manuella Rey for her excellent technical assistance. This work was supported by Swiss National Science Foundation grant no. 3100A0-100797/1 (to AC) and the Ruth Mortimer Bequest (to PF). The authors declare that there is no conflict of interest that would prejudice the impartiality of this scientific work.

\section{References}

Babu PS, Bavers DL, Beuschlein F, Shah S, Jeffs B, Jameson JL \& Hammer GD 2002 Interaction between Dax-1 and steroidogenic factor-1 in vivo: increased adrenal responsiveness to ACTH in the absence of Dax-1. Endocrinology 143 665-673.

Bakke M \& Lund J 1995 Transcriptional regulation of the bovine CYP17 gene: two nuclear orphan receptors determine activity of cAMP-responsive sequence 2. Endocrine Research 21 509-516.

Brand E, Chatelain N, Mulatero P, Fery I, Curnow K, Jeunemaitre X, Corvol P, Pascoe L \& Soubrier F 1998 Structural analysis and evaluation of the aldosterone synthase gene in hypertension. Hypertension 32 198-204. 
Buholzer CF, Arrighi JF, Abraham S, Piguet V, Capponi AM \& Casal AJ 2005 Chicken ovalbumin upstream promoter-transcription factor (COUP-TF) is a negative regulator of steroidogenesis in bovine adrenal glomerulosa cells. Molecular Endocrinology 19 65-75.

Caron KM, Ikeda Y, Soo SC, Stocco DM, Parker KL \& Clark BJ 1997 Characterization of the promoter region of the mouse gene encoding the steroidogenic acute regulatory protein. Molecular Endocrinology 11 138-147.

Clark BJ, Wells J, King SR \& Stocco DM 1994 The purification, cloning, and expression of a novel luteinizing hormone-induced mitochondrial protein in MA-10 mouse Leydig tumor cells. Characterization of the steroidogenic acute regulatory protein (StAR). Journal of Biological Chemistry 269 28314-28322.

Cooney AJ, Lee CT, Lin SC, Tsai SY \& Tsai MJ 2001 Physiological function of the orphans GCNF and COUP-TF. Trends in Endocrinology and Metabolism 12 247-251.

Crivello JF \& Jefcoate CR 1980 Intracellular movement of cholesterol in rat adrenal cells. Kinetics and effects of inhibitors. Journal of Biological Chemistry 255 8144-8151.

Davies E, Holloway CD, Ingram MC, Inglis GC, Friel EC, Morrison C, Anderson NH, Fraser R \& Connell JM 1999 Aldosterone excretion rate and blood pressure in essential hypertension are related to polymorphic differences in the aldosterone synthase gene CYP11B2. Hypertension 33 703-707.

Ferrari P, Shaw SG, Nicod J, Saner E \& Nussberger J 2004 Active renin versus plasma renin activity to define aldosterone-to-renin ratio for primary aldosteronism. Journal of Hypertension 22 377-381.

Garbers DL \& Dubois SK 1999 The molecular basis of hypertension. Annual Review of Biochemistry 68 127-155.

Hiroi H, Christenson LK, Chang L, Sammel MD, Berger SL \& Strauss JF 3rd 2004 Temporal and spatial changes in transcription factor binding and histone modifications at the steroidogenic acute regulatory protein (StAR) locus associated with StAR transcription. Molecular Endocrinology 18 791-806.

Ito M, Yu R \& Jameson JL 1997 DAX-1 inhibits SF-1-mediated transactivation via a carboxy-terminal domain that is deleted in adrenal hypoplasia congenita. Molecular and Cellular Biology 17 1476-1483.

Lalli E, Melner MH, Stocco DM \& Sassone-Corsi P 1998 DAX-1 blocks steroid production at multiple levels. Endocrinology 139 4237-4243.

Lim PO, Macdonald TM, Holloway C, Friel E, Anderson NH, Dow E, Jung RT, Davies E, Fraser R \& Connell JM 2002 Variation at the aldosterone synthase (CYP11B2) locus contributes to hypertension in subjects with a raised aldosterone-to-renin ratio. Journal of Clinical Endocrinology and Metabolism 87 4398-4402.

Lin D, Sugawara T, Strauss JF 3rd, Clark BJ, Stocco DM, Saenger P, Rogol A \& Miller WL 1995 Role of steroidogenic acute regulatory protein in adrenal and gonadal steroidogenesis. Science $\mathbf{2 6 7}$ 1828-1831.

Lovati E, Richard A, Frey BM, Frey FJ \& Ferrari P 2001 Genetic polymorphisms of the renin-angiotensin-aldosterone system in end-stage renal disease. Kidney International 60 46-54.

Manna PR, Wang XJ \& Stocco DM 2003 Involvement of multiple transcription factors in the regulation of steroidogenic acute regulatory protein gene expression. Steroids 68 1125-1134.

Miller WL 1997 Congenital lipoid adrenal hyperplasia: the human gene knockout for the steroidogenic acute regulatory protein. Journal of Molecular Endocrinology 19 227-240.

Monte D, DeWitte F \& Hum DW 1998 Regulation of the human $\mathrm{P} 450$ scc gene by steroidogenic factor 1 is mediated by CBP/p300. Journal of Biological Chemistry 273 4585-4591.

Nicod J, Bruhin D, Auer L, Vogt B, Frey FJ \& Ferrari P 2003 A biallelic gene polymorphism of CYP11B2 predicts increased aldosterone to renin ratio in selected hypertensive patients. Journal of Clinical Endocrinology and Metabolism 88 2495-2500.
Osman H, Murigande C, Nadakal A \& Capponi AM 2002 Repression of DAX-1 and induction of SF-1 expression. Two mechanisms contributing to the activation of aldosterone biosynthesis in adrenal glomerulosa cells. Journal of Biological Chemistry 277 41259-41267.

Paillard F, Chansel D, Brand E, Benetos A, Thomas F, Czekalski S, Ardaillou R \& Soubrier F 1999 Genotype-phenotype relationships for the renin-angiotensin-aldosterone system in a normal population. Hypertension 34 423-429.

Privalle CT, Crivello JF \& Jefcoate CR 1983 Regulation of intramitochondrial cholesterol transfer to side-chain cleavage cytochrome P-450 in rat adrenal gland. PNAS 80 702-706.

Reinhart AJ, Williams SC, Clark BJ \& Stocco DM 1999 SF-1 (steroidogenic factor-1) and C/EBP beta (CCAAT/enhancer binding protein-beta) cooperate to regulate the murine StAR (steroidogenic acute regulatory) promoter. Molecular Endocrinology 13 729-741.

Sandhoff TW, Hales DB, Hales KH \& McLean MP 1998 Transcriptional regulation of the rat steroidogenic acute regulatory protein gene by steroidogenic factor 1. Endocrinology 139 4820-4831.

Shibata H, Ikeda Y, Mukai T, Morohashi K, Kurihara I, Ando T, Suzuki T, Kobayashi S, Murai M \& Saito I 2001 Expression profiles of COUP-TF, DAX-1, and SF-1 in the human adrenal gland and adrenocortical tumors: possible implications in steroidogenesis. Molecular Genetics and Metabolism 74 206-216.

Shibata H, Kurihara I, Kobayashi S, Yokota K, Suda N, Saito I \& Saruta T 2003 Regulation of differential COUP-TF-coregulator interactions in adrenal cortical steroidogenesis. Journal of Steroid Biochemistry and Molecular Biology 85 449-456.

Stocco DM 2001 Tracking the role of a star in the sky of the new millennium. Molecular Endocrinology 15 1245-1254.

Sugawara T, Kiriakidou M, McAllister JM, Kallen CB \& Strauss JF 3rd 1997 Multiple steroidogenic factor 1 binding elements in the human steroidogenic acute regulatory protein gene $5^{\prime}$-flanking region are required for maximal promoter activity and cyclic AMP responsiveness. Biochemistry 36 7249-7255.

Sugawara T, Saito M \& Fujimoto S 2000 Sp1 and SF-1 interact and cooperate in the regulation of human steroidogenic acute regulatory protein gene expression. Endocrinology 141 2895-2903.

Suzuki T, Kasahara M, Yoshioka H, Morohashi K \& Umesono K 2003 LXXLL-related motifs in Dax-1 have target specificity for the orphan nuclear receptors Ad4BP/SF-1 and LRH-1. Molecular and Cellular Biology 23 238-249.

Takeda Y, Furukawa K, Inaba S, Miyamori I \& Mabuchi H 1999 Genetic analysis of aldosterone synthase in patients with idiopathic hyperaldosteronism. Journal of Clinical Endocrinology and Metabolism 84 1633-1637.

White PC \& Slutsker L 1995 Haplotype analysis of CYP11B2. Endocrine Research 21 437-442.

Zazopoulos E, Lalli E, Stocco DM \& Sassone-Corsi P 1997 DNA binding and transcriptional repression by DAX-1 blocks steroidogenesis. Nature 390 311-315.

Zeitoun K, Takayama K, Michael MD \& Bulun SE 1999 Stimulation of aromatase $\mathrm{P} 450$ promoter (II) activity in endometriosis and its inhibition in endometrium are regulated by competitive binding of steroidogenic factor-1 and chicken ovalbumin upstream promoter transcription factor to the same cis-acting element. Molecular Endocrinology 13 239-253.

Zhang Y \& Dufau ML 2003 Dual mechanisms of regulation of transcription of luteinizing hormone receptor gene by nuclear orphan receptors and histone deacetylase complexes. Journal of Steroid Biochemistry and Molecular Biology 85 401-414.

Received in final form 20 April 2006 Accepted 21 April 2006

Made available online as an Accepted Preprint 4 May 2006 